\title{
PTF 10fqs: A LUMINOUS RED NOVA IN THE SPIRAL GALAXY MESSIER 99
}

\author{
Mansi M. Kasliwal ${ }^{1}$, Shri R. Kulkarni ${ }^{1}$, Iair Arcavi ${ }^{2}$, Robert M. Quimby ${ }^{1}$, Eran O. Ofek ${ }^{1}$, Peter Nugent ${ }^{3}$, \\ Janet Jacobsen ${ }^{3}$, Avishay Gal-Yam ${ }^{2}$, Yoav Green ${ }^{2}$, Ofer Yaron ${ }^{2}$, Derek B. Fox ${ }^{4}$, Jacob L. Howell ${ }^{4}$, S. Bradley Cenko ${ }^{5}$, \\ Io Kleiser ${ }^{5}$, Joshua S. Bloom ${ }^{5}$, Adam Miller ${ }^{5}$, Weidong Li ${ }^{5}$, Alexei V. Filippenko ${ }^{5}$, Dan Stark ${ }^{5}$, Dovi Poznanski ${ }^{3,5}$, \\ Nicholas M. Law ${ }^{6}$, George Helou ${ }^{7}$, Dale A. Frail ${ }^{8}$, James D. Neill ${ }^{1}$, Karl Forster ${ }^{1}$, D. Christopher Martin ${ }^{1}$, \\ Shriharsh P. Tendulkar ${ }^{1}$, Neil Gehrels ${ }^{9}$, Jamie Kennea ${ }^{10}$, Mark Sullivan ${ }^{11}$, Lars Bildsten $^{12,13}$, Richard DeKany ${ }^{14}$, \\ Gustavo Rahmer ${ }^{14}$, David Hale ${ }^{14}$, Roger Smith ${ }^{14}$, JefF Zolkower ${ }^{14}$, Viswa Velur ${ }^{14}$, Richard Walters ${ }^{14}$, \\ John Henning ${ }^{14}$, Kahnh Bui ${ }^{14}$, Dan McKenna ${ }^{14}$, And Cullen BlaKe ${ }^{15}$ \\ ${ }^{1}$ Cahill Center for Astrophysics, California Institute of Technology, Pasadena, CA 91125, USA \\ ${ }^{2}$ Benoziyo Center for Astrophysics, Faculty of Physics, The Weizmann Institute of Science, Rehovot 76100, Israel \\ ${ }^{3}$ Computational Cosmology Center, Lawrence Berkeley National Laboratory, 1 Cyclotron Road, Berkeley, CA 94720, USA \\ ${ }^{4}$ Astronomy and Astrophysics, Eberly College of Science, The Pennsylvania State University, University Park, PA 16802, USA \\ ${ }^{5}$ Department of Astronomy, University of California, Berkeley, CA 94720-3411, USA \\ ${ }^{6}$ Dunlap Institute for Astronomy and Astrophysics, University of Toronto, 50 St. George Street, Toronto M5S 3H4, Ontario, Canada \\ ${ }^{7}$ Infrared Processing and Analysis Center, California Institute of Technology, Pasadena, CA 91125, USA \\ ${ }^{8}$ National Radio Astronomy Observatory, Array Operations Center, Socorro, NM 87801, USA \\ ${ }^{9}$ NASA-Goddard Space Flight Center, Greenbelt, MD 20771, USA \\ ${ }^{10}$ Department of Astronomy and Astrophysics, Pennsylvania State University, State College, PA 16802, USA \\ ${ }^{11}$ Department of Physics, Oxford University, Oxford OX1 3RH, UK \\ 12 Kavli Institute of Theoretical Physics, University of California Santa Barbara, Santa Barbara, CA 93106, USA \\ ${ }^{13}$ Department of Physics, University of California Santa Barbara, Santa Barbara, CA 93106, USA \\ ${ }^{14}$ Caltech Optical Observatories, California Institute of Technology, Pasadena, CA 91125, USA \\ ${ }^{15}$ Department of Astrophysical Sciences, Princeton University, Princeton, NJ 08544, USA \\ Received 2010 July 20; accepted 2011 February 4; published 2011 March 14
}

\begin{abstract}
The Palomar Transient Factory (PTF) is systematically charting the optical transient and variable sky. A primary science driver of PTF is building a complete inventory of transients in the local universe (distance less than $200 \mathrm{Mpc}$ ). Here, we report the discovery of PTF 10fqs, a transient in the luminosity "gap" between novae and supernovae. Located on a spiral arm of Messier 99, PTF 10fqs has a peak luminosity of $M_{r}=-12.3$, red color $(g-r=1.0)$, and is slowly evolving (decayed by $1 \mathrm{mag}$ in 68 days). It has a spectrum dominated by intermediate-width $\mathrm{H} \alpha$ $\left(\approx 930 \mathrm{~km} \mathrm{~s}^{-1}\right)$ and narrow calcium emission lines. The explosion signature (the light curve and spectra) is overall similar to that of M85 OT2006-1, SN 2008S, and NGC 300 OT. The origin of these events is shrouded in mystery and controversy (and in some cases, in dust). PTF 10fqs shows some evidence of a broad feature (around $8600 \AA$ ) that may suggest very large velocities $\left(\approx 10,000 \mathrm{~km} \mathrm{~s}^{-1}\right)$ in this explosion. Ongoing surveys can be expected to find a few such events per year. Sensitive spectroscopy, infrared monitoring, and statistics (e.g., disk versus bulge) will eventually make it possible for astronomers to unravel the nature of these mysterious explosions.
\end{abstract}

Key words: stars: AGB and post-AGB - stars: mass-loss - supernovae: general - supernovae: individual (PTF 10fqs) - surveys

Online-only material: color figures

\section{INTRODUCTION}

Two reasons motivate us to search for transients in the local universe (distance $<200 \mathrm{Mpc}$ ). First, the emerging areas of gravitational wave astronomy, high-energy cosmic rays, very high energy photons, and neutrino astronomy are limited to this distance horizon either due to physical effects (optical depth) or instrumental sensitivity. Thus, to effectively search for an electromagnetic analog, understanding the full range of transient phenomena is essential. For instance, the electromagnetic counterpart to the gravitational wave signature of neutron star mergers is expected to be fainter and faster than that of supernovae (e.g., Metzger et al. 2010).

Our second motivation is one of pure exploration. The peak luminosity of novae ranges between -4 and $-10 \mathrm{mag},{ }^{16}$ whereas supernovae range between -15 and -22 mag. The

\footnotetext{
${ }^{16}$ Unless explicitly noted, quoted magnitudes are in the $R$ band.
}

large gap between the cataclysmic novae and the catastrophic supernovae has been noted by early observers. Theorists have proposed several intriguing scenarios producing transients in this "gap" (e.g., Bildsten et al. 2007; Metzger et al. 2009; Shen et al. 2010; Moriya et al. 2010).

The Palomar Transient Factory ${ }^{17}$ (PTF; see Rahmer et al. 2008; Law et al. 2009; Rau et al. 2009) was designed to undertake a systematic exploration of the transient sky in the optical bands. One of the key projects of PTF is to build a complete inventory of transients in the local universe. PTF has a "Dynamic" cadence experiment which undertakes frequent observations of fields, optimized for inclusion of galaxies in the local universe. A description of the design sensitivity is given elsewhere (Kulkarni \& Kasliwal 2009). Here, we report on the discovery of PTF 10fqs, a transient in this "gap" between novae and supernovae.

17 http://www.astro.caltech.edu/ptf. 


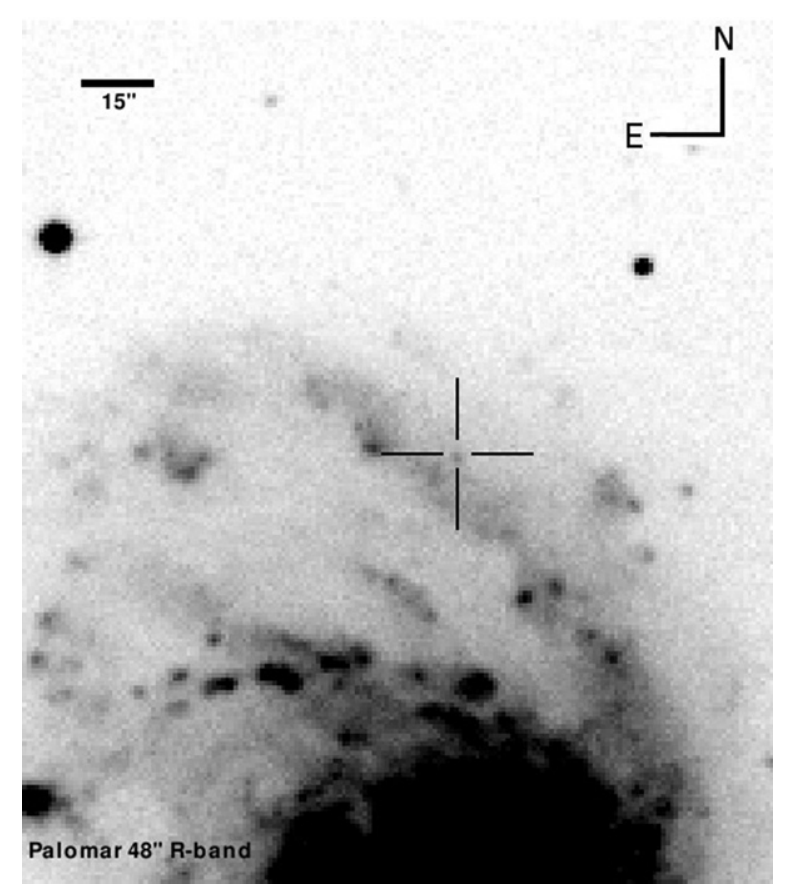

Figure 1. Discovery image of PTF 10fqs (obtained with the Palomar Oschin 48 inch telescope on 2010 April 16.393). The transient is marked by a cross and located at $\alpha(\mathrm{J} 2000)=12^{\mathrm{h}} 18^{\mathrm{m}} 50^{\mathrm{s}} .16$ and $\delta(\mathrm{J} 2000)=+14^{\circ} 26^{\prime} 39^{\prime \prime} \cdot 2$. With respect to the host-galaxy nucleus, the transient is offset by 8." $1 \mathrm{E}$ and $99 . \prime 9 \mathrm{~N}$.

\section{DISCOVERY}

On 2010 April 16.393 (UT dates are used throughout this paper), the PTF discovered an optical transient toward Messier 99 (M99; see Figure 1). Following the PTF discovery naming sequence, this transient was dubbed PTF 10fqs and reported via an ATEL (Kasliwal \& Kulkarni 2010).

M99 (NGC 4254), ${ }^{18}$ an Sc galaxy, is one of the brighter spiral members of the Virgo Cluster. The recession velocity of the galaxy is about $2400 \mathrm{~km} \mathrm{~s}^{-1}$. Over the past 50 years, three supernovae have been discovered in this galaxy: SN 1967H (Type II?, Fairall 1972), SN 1972Q (Type II; Barbon et al. 1973), ${ }^{19}$ and SN 1986I (Type II; Pennypacker et al. 1989).

At discovery, the brightness of PTF 10fqs was $R=20.0 \pm$ 0.2 mag. There are no previous detections in PTF data taken on and prior to April 10. If located in M99, the absolute magnitude (for an assumed distance of $17 \mathrm{Mpc}$; Russell 2002) corresponds to $M_{R}=-11.1$. We concluded that the object could be (in decreasing order of probability) a foreground variable star, a young supernova, or a transient in the "gap." These possibilities can be easily distinguished by spectroscopic observations.

\section{FOLLOW-UP OBSERVATIONS}

\subsection{Spectra}

We triggered our Target-of-Opportunity (TOO) program on the $8 \mathrm{~m}$ Gemini-South telescope. On 2010 April 18.227, the Gemini Observatory staff observed PTF 10fqs with the Gemini Multi-Object Spectrograph (GMOS; Hook et al. 2004). The parameters for the observations were: R400 grating, orderblocking filter GG455_G039, and a 1".0 slit. Two 10 minute

\footnotetext{
18 http://seds.org/messier/m/m099.html.

19 Curiously, the reported position of SN 1972Q was only 3.' 6 from PTF 10fqs. We did a careful registration of the discovery image of SN 1972Q (Barbon et al. 1973) and PTF $10 \mathrm{fqs}$ and find that the offset is actually $11^{\prime \prime} .0 \mathrm{E}, 0^{\prime \prime} 8 \mathrm{~S}$.
}

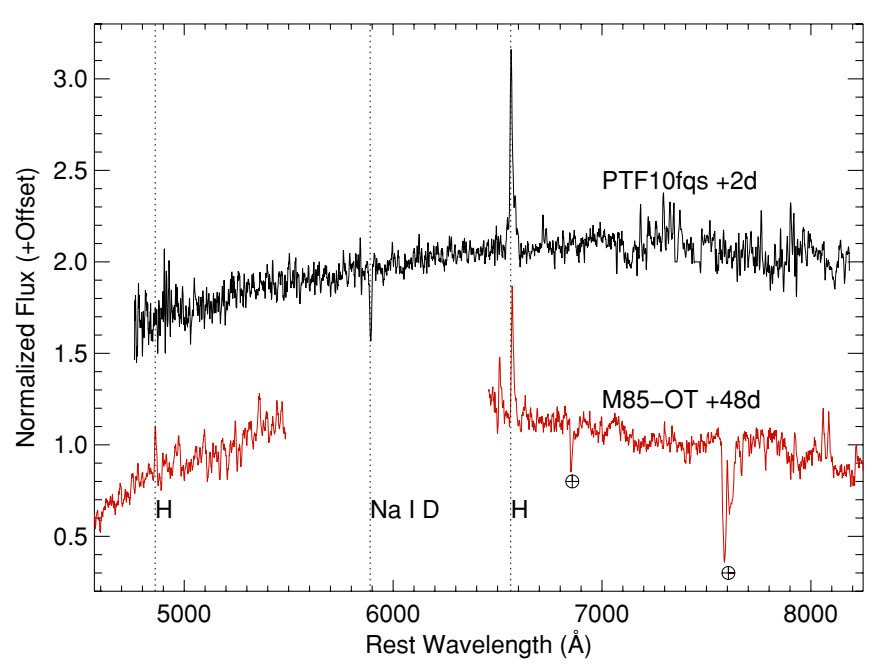

Figure 2. Gemini GMOS spectrum of PTF 10fqs (black) taken two days after discovery. The wavelength coverage is continuous over the range 4600-8800 $\AA$. The most prominent emission feature is $\mathrm{H} \alpha$. Plotted below for comparison, the spectrum of M85OT-2006-1 (red; Kulkarni et al. 2007).

(A color version of this figure is available in the online journal.)

integrations centered on 6700 and $6800 \AA$ A were obtained. The two observations allowed for coverage of the gap between the chips. The package gemini gmos working in the iraf framework was used to reduce the data. The spectrum is shown in Figure 2.

The most prominent emission feature is an intermediate width $\left(13 \AA, 600 \mathrm{~km} \mathrm{~s}^{-1}\right)^{20} \mathrm{H} \alpha$ line consistent with the recession velocity of the galaxy $\left(2400 \mathrm{~km} \mathrm{~s}^{-1}\right.$; see below). $\mathrm{H} \beta$ was not detected. From this spectrum alone, we concluded that PTF 10fqs is in M99 and the intermediate line width made it unlikely to be a supernova. PTF 10 fqs appeared to be a transient in the "gap," and we initiated extensive multi-band follow-up observations.

We continued to monitor the spectral evolution with the Marcario Low-Resolution Spectrograph (LRS; Hill et al. 1998) on the Hobby-Eberly Telescope (HET) ${ }^{21}$ We used the G1 grating, with a $2^{\prime \prime}$ slit and a GG385 order-blocking filter, providing resolution $R=\lambda / \Delta \lambda \approx 360$ over 4200-9200 A. Data were reduced using the onedspec package in the iraf environment, with cosmic-ray rejection via the la_cosmic package (van Dokkum 2001), and with spectrophotometric corrections applied using standard-star observations (specifically, BD332642).

On May 15, we also obtained relatively higher resolution spectroscopic observations and relatively better blue coverage with the Low-Resolution Imaging Spectrograph (Oke et al. 1995 ) on the Keck I telescope. First, we used the $831 / 8200$ grating centered on $7905 \AA$ to get higher resolution spectra of the calcium lines. On the blue side, we used the 300/5000 grism to cover $\mathrm{Ca} \mathrm{H}+\mathrm{K}$ lines. For higher resolution covering the Balmer lines, we used the $600 / 7500$ grating (centered on $7201 \AA$ ) in conjunction with the $600 / 4000$ grism.

The log of spectroscopic observations is given in Table 1. The spectral evolution is shown in Figure 3.

\footnotetext{
${ }^{20}$ The velocity quoted here is corrected for instrumental resolution and is measured as the Gaussian full width at half-maximum (GFWHM) of the emission line.

${ }^{21}$ Director's Discretionary Time; PI: D. Fox.
} 


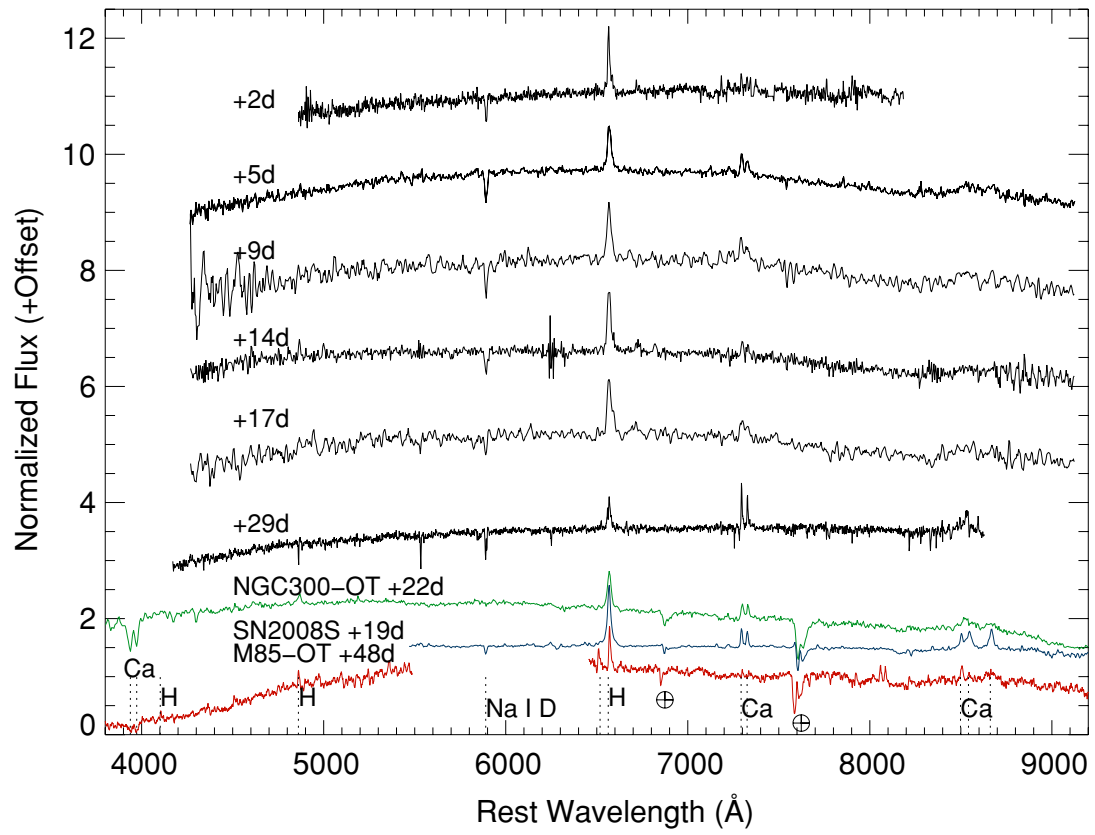

Figure 3. Spectra of PTF 10fqs at various epochs (phase in days is defined relative to discovery epoch). Also shown are spectra of NGC 300-OT (Bond et al. 2009), M85OT2006-1 (Kulkarni et al. 2007), and SN2008S (Botticella et al. 2009). The wavelength has been corrected for the recession velocity of each galaxy $(z=0.0024$ for M85, $z=0.008$ for M99, $z=0.00048$ for NGC 300, and $z=0.00016$ for NGC 6946).

Table 1

Log of Spectroscopic Observations

\begin{tabular}{lcclc}
\hline \hline Date (UT 2010) & MJD & \multicolumn{1}{c}{ Exposure } & \multicolumn{1}{c}{ Facility } & Grating/Grism \\
\hline Apr 18.23 & 55304.23 & $2 \times 600 \mathrm{~s}$ & Gemini-S/GMOS & 400 \\
Apr 21.31 & 55307.31 & $2 \times 800 \mathrm{~s}$ & HET/LRS & 360 \\
Apr 25.29 & 55311.29 & $2 \times 600 \mathrm{~s}$ & HET/LRS & 360 \\
Apr 30.12 & 55316.12 & $2 \times 600 \mathrm{~s}$ & HET/LRS & 360 \\
May 3.28 & 55319.28 & $2 \times 600 \mathrm{~s}$ & HET/LRS & 360 \\
May 15.26 & 55331.26 & $3 \times 600 \mathrm{~s}$ & Keck I/LRIS & 831 \\
May 15.26 & 55331.26 & $1 \times 2000 \mathrm{~s}$ & Keck I/LRIS & 300 \\
May 15.31 & 55331.31 & $3 \times 650 \mathrm{~s}$ & Keck I/LRIS & 600 \\
\hline
\end{tabular}

\subsection{Optical and Near-infrared Imaging}

Observations with the robotic Palomar 60 inch telescope (Cenko et al. 2006) on April 20.4 confirmed that PTF 10fqs was rising $(r=19.4 \pm 0.1 \mathrm{mag})$ and red $(g-r=1.0 \mathrm{mag})$. We show the photometric evolution in gri bands in Figure 4 and Table 2. On April 27.2, the light curve peaked at $r=18.9 \pm 0.1 \mathrm{mag}$ corresponding to $M_{r}=-12.3$ (correcting for foreground Galactic extinction of $E(B-V)=0.039$; Schlegel et al. 1998). Aperture photometry was done after image subtraction using a custom modification of the CPM algorithm, mkdifflc (Gal-Yam et al. 2004). Template images for subtraction and reference magnitudes for zero-point computation were taken from the Sloan Digital Sky Survey (Abazajian et al. 2009).

Near-infrared images were obtained with the Peters Automated Infrared Imaging Telescope (PAIRITEL; Bloom et al. 2006), and reduced by an automated reduction pipeline. We lack sufficiently deep template images, which are free of light from PTF 10fqs, to perform reliable image subtraction. Thus, we measure the flux from the source in a small circular aperture, removing the sky with a nearby background region, and adopt a systematic error of $0.2 \mathrm{mag}$ in the $J$ and $H$ bands and $0.3 \mathrm{mag}$ in the $K_{s}$ band. The values reported in Table 2 have been

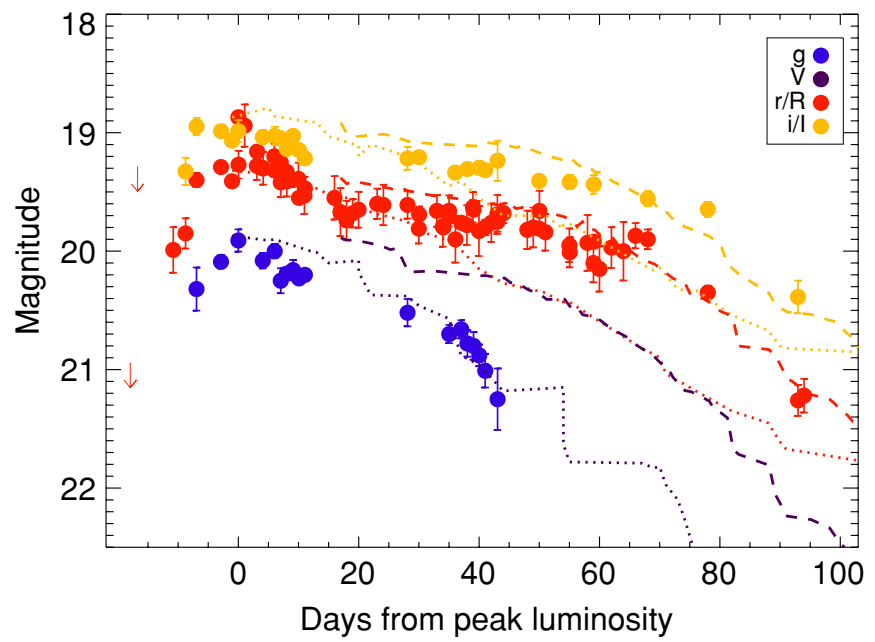

Figure 4. Multi-band light curve of PTF 10fqs obtained with the Palomar 48 inch (squares) and Palomar 60 inch (circles) telescopes. Upper limits are denoted by downward arrows. Note that the evolution is relatively faster in the $g$ band compared to the $r$ band. Also shown for comparison are the VRI-band light curves of SN2008S (dotted; Botticella et al. 2009) and NGC 300-OT (dashed; Bond et al. 2009). The light curves are shifted vertically by a constant ( +3 mag for SN2008S and +5.2 mag for NGC 300-OT) such that their $R$-band light curves are at the same level as the $r$-band light curve of PTF 10fqs.

calibrated against the Two Micron All Sky Survey (2MASS) system (Cohen et al. 2003).

\subsection{Radio Observations}

We observed PTF 10fqs with the EVLA on April 20.19-20.26 at central frequencies of $4.96 \mathrm{GHz}$ and $8.46 \mathrm{GHz}$. We added together two adjacent $128 \mathrm{MHz}$ subbands with full polarization to maximize continuum sensitivity. Amplitude and bandpass calibration was achieved using a single observation of $\mathrm{J} 1331+3030$, and phase calibration was carried out every 
Table 2

Optical and Near-infrared Light Curve

\begin{tabular}{|c|c|c|c|}
\hline Date (MJD) & Filter & Mag & Facility \\
\hline 55295.2 & Mould-R & $>20.94$ & Palomar 48 inch \\
\hline 55296.5 & Mould-R & $>19.28$ & Palomar 48 inch \\
\hline 55302.4 & Mould-R & $19.99 \pm 0.19$ & Palomar 48 inch \\
\hline 55313.2 & Mould-R & $19.27 \pm 0.11$ & Palomar 48 inch \\
\hline 55316.3 & Mould-R & $19.28 \pm 0.11$ & Palomar 48 inch \\
\hline 55317.3 & Mould-R & $19.30 \pm 0.13$ & Palomar 48 inch \\
\hline 55319.2 & Mould-R & $19.20 \pm 0.10$ & Palomar 48 inch \\
\hline 55320.2 & Mould-R & $19.42 \pm 0.12$ & Palomar 48 inch \\
\hline 55321.3 & Mould-R & $19.41 \pm 0.12$ & Palomar 48 inch \\
\hline 55323.2 & Mould-R & $19.39 \pm 0.13$ & Palomar 48 inch \\
\hline 55324.2 & Mould-R & $19.53 \pm 0.15$ & Palomar 48 inch \\
\hline 55329.2 & Mould-R & $19.55 \pm 0.18$ & Palomar 48 inch \\
\hline 55330.2 & Mould-R & $19.67 \pm 0.20$ & Palomar 48 inch \\
\hline 55331.2 & Mould-R & $19.74 \pm 0.16$ & Palomar 48 inch \\
\hline 55332.2 & Mould-R & $19.68 \pm 0.11$ & Palomar 48 inch \\
\hline 55333.2 & Mould-R & $19.65 \pm 0.15$ & Palomar 48 inch \\
\hline 55336.3 & Mould-R & $19.60 \pm 0.12$ & Palomar 48 inch \\
\hline 55337.3 & Mould-R & $19.61 \pm 0.17$ & Palomar 48 inch \\
\hline 55343.2 & Mould-R & $19.81 \pm 0.12$ & Palomar 48 inch \\
\hline 55346.2 & Mould-R & $19.66 \pm 0.13$ & Palomar 48 inch \\
\hline 55347.2 & Mould-R & $19.79 \pm 0.17$ & Palomar 48 inch \\
\hline 55348.2 & Mould-R & $19.66 \pm 0.13$ & Palomar 48 inch \\
\hline 55349.3 & Mould-R & $19.90 \pm 0.19$ & Palomar 48 inch \\
\hline 55351.2 & Mould-R & $19.78 \pm 0.16$ & Palomar 48 inch \\
\hline 55352.2 & Mould-R & $19.63 \pm 0.12$ & Palomar 48 inch \\
\hline 55353.2 & Mould-R & $19.83 \pm 0.21$ & Palomar 48 inch \\
\hline 55355.2 & Mould-R & $19.76 \pm 0.16$ & Palomar 48 inch \\
\hline 55356.2 & Mould-R & $19.69 \pm 0.16$ & Palomar 48 inch \\
\hline 55361.2 & Mould-R & $19.82 \pm 0.16$ & Palomar 48 inch \\
\hline 55362.2 & Mould-R & $19.80 \pm 0.16$ & Palomar 48 inch \\
\hline 55363.2 & Mould-R & $19.66 \pm 0.16$ & Palomar 48 inch \\
\hline 55364.2 & Mould-R & $19.84 \pm 0.15$ & Palomar 48 inch \\
\hline 55368.2 & Mould-R & $19.95 \pm 0.14$ & Palomar 48 inch \\
\hline 55371.2 & Mould-R & $19.93 \pm 0.23$ & Palomar 48 inch \\
\hline 55372.2 & Mould-R & $20.10 \pm 0.16$ & Palomar 48 inch \\
\hline 55373.2 & Mould-R & $20.15 \pm 0.19$ & Palomar 48 inch \\
\hline 55375.2 & Mould-R & $19.97 \pm 0.17$ & Palomar 48 inch \\
\hline 55377.2 & Mould-R & $20.00 \pm 0.24$ & Palomar 48 inch \\
\hline 55379.2 & Mould-R & $19.87 \pm 0.10$ & Palomar 48 inch \\
\hline 55304.4 & $r$ & $19.85 \pm 0.12$ & Palomar 60 inch \\
\hline 55306.3 & $r$ & $19.40 \pm 0.05$ & Palomar 60 inch \\
\hline 55310.3 & $r$ & $19.29 \pm 0.03$ & Palomar 60 inch \\
\hline 55312.1 & $r$ & $19.41 \pm 0.03$ & Palomar 60 inch \\
\hline 55313.2 & $r$ & $18.87 \pm 0.05$ & Palomar 60 inch \\
\hline 55314.2 & $r$ & $18.94 \pm 0.17$ & Palomar 60 inch \\
\hline 55316.3 & $r$ & $19.16 \pm 0.05$ & Palomar 60 inch \\
\hline 55317.3 & $r$ & $19.30 \pm 0.05$ & Palomar 60 inch \\
\hline 55319.2 & $r$ & $19.32 \pm 0.04$ & Palomar 60 inch \\
\hline 55320.2 & $r$ & $19.25 \pm 0.01$ & Palomar 60 inch \\
\hline 55321.2 & $r$ & $19.33 \pm 0.02$ & Palomar 60 inch \\
\hline 55322.3 & $r$ & $19.40 \pm 0.02$ & Palomar 60 inch \\
\hline 55323.3 & $r$ & $19.55 \pm 0.04$ & Palomar 60 inch \\
\hline 55324.3 & $r$ & $19.47 \pm 0.02$ & Palomar 60 inch \\
\hline 55341.3 & $r$ & $19.61 \pm 0.11$ & Palomar 60 inch \\
\hline 55343.2 & $r$ & $19.69 \pm 0.06$ & Palomar 60 inch \\
\hline 55347.3 & $r$ & $19.80 \pm 0.04$ & Palomar 60 inch \\
\hline 55348.2 & $r$ & $19.71 \pm 0.01$ & Palomar 60 inch \\
\hline 55350.2 & $r$ & $19.76 \pm 0.03$ & Palomar 60 inch \\
\hline 55352.3 & $r$ & $19.65 \pm 0.03$ & Palomar 60 inch \\
\hline 55354.2 & $r$ & $19.80 \pm 0.06$ & Palomar 60 inch \\
\hline 55356.3 & $r$ & $19.75 \pm 0.08$ & Palomar 60 inch \\
\hline 55357.3 & $r$ & $19.68 \pm 0.08$ & Palomar 60 inch \\
\hline 55363.2 & $r$ & $19.81 \pm 0.03$ & Palomar 60 inch \\
\hline 55368.3 & $r$ & $20.01 \pm 0.12$ & Palomar 60 inch \\
\hline 55372.2 & $r$ & $19.92 \pm 0.03$ & Palomar 60 inch \\
\hline
\end{tabular}

Table 2

(Continued)

\begin{tabular}{|c|c|c|c|}
\hline Date (MJD) & Filter & Mag & Facility \\
\hline 55381.2 & $r$ & $19.90 \pm 0.08$ & Palomar 60 inch \\
\hline 55391.2 & $r$ & $20.35 \pm 0.05$ & Palomar 60 inch \\
\hline 55406.2 & $r$ & $21.26 \pm 0.13$ & Palomar 60 inch \\
\hline 55407.2 & $r$ & $21.22 \pm 0.14$ & Palomar 60 inch \\
\hline 55306.3 & $g$ & $20.32 \pm 0.18$ & Palomar 60 inch \\
\hline 55310.3 & $g$ & $20.09 \pm 0.05$ & Palomar 60 inch \\
\hline 55313.2 & $g$ & $19.91 \pm 0.09$ & Palomar 60 inch \\
\hline 55317.3 & $g$ & $20.08 \pm 0.06$ & Palomar 60 inch \\
\hline 55319.2 & $g$ & $20.00 \pm 0.06$ & Palomar 60 inch \\
\hline 55320.2 & $g$ & $20.25 \pm 0.10$ & Palomar 60 inch \\
\hline 55321.2 & $g$ & $20.19 \pm 0.03$ & Palomar 60 inch \\
\hline 55322.3 & $g$ & $20.16 \pm 0.08$ & Palomar 60 inch \\
\hline 55323.3 & $g$ & $20.23 \pm 0.04$ & Palomar 60 inch \\
\hline 55324.3 & $g$ & $20.20 \pm 0.02$ & Palomar 60 inch \\
\hline 55341.3 & $g$ & $20.52 \pm 0.11$ & Palomar 60 inch \\
\hline 55348.2 & $g$ & $20.70 \pm 0.07$ & Palomar 60 inch \\
\hline 55350.2 & $g$ & $20.66 \pm 0.07$ & Palomar 60 inch \\
\hline 55351.3 & $g$ & $20.78 \pm 0.11$ & Palomar 60 inch \\
\hline 55352.3 & $g$ & $20.80 \pm 0.11$ & Palomar 60 inch \\
\hline 55353.2 & $g$ & $20.88 \pm 0.09$ & Palomar 60 inch \\
\hline 55354.2 & $g$ & $21.01 \pm 0.14$ & Palomar 60 inch \\
\hline 55356.3 & $g$ & $21.25 \pm 0.25$ & Palomar 60 inch \\
\hline 55304.4 & $i$ & $19.32 \pm 0.11$ & Palomar 60 inch \\
\hline 55306.3 & $i$ & $18.94 \pm 0.07$ & Palomar 60 inch \\
\hline 55310.3 & $i$ & $18.98 \pm 0.03$ & Palomar 60 inch \\
\hline 55312.2 & $i$ & $19.06 \pm 0.04$ & Palomar 60 inch \\
\hline 55313.2 & $i$ & $18.98 \pm 0.09$ & Palomar 60 inch \\
\hline 55317.3 & $i$ & $19.03 \pm 0.06$ & Palomar 60 inch \\
\hline 55319.2 & $i$ & $19.02 \pm 0.07$ & Palomar 60 inch \\
\hline 55320.2 & $i$ & $19.04 \pm 0.03$ & Palomar 60 inch \\
\hline 55321.2 & $i$ & $19.13 \pm 0.03$ & Palomar 60 inch \\
\hline 55322.3 & $i$ & $19.02 \pm 0.04$ & Palomar 60 inch \\
\hline 55323.3 & $i$ & $19.14 \pm 0.03$ & Palomar 60 inch \\
\hline 55324.2 & $i$ & $19.21 \pm 0.04$ & Palomar 60 inch \\
\hline 55341.3 & $i$ & $19.21 \pm 0.09$ & Palomar 60 inch \\
\hline 55343.2 & $i$ & $19.20 \pm 0.02$ & Palomar 60 inch \\
\hline 55349.2 & $i$ & $19.33 \pm 0.02$ & Palomar 60 inch \\
\hline 55351.3 & $i$ & $19.30 \pm 0.03$ & Palomar 60 inch \\
\hline 55353.2 & $i$ & $19.29 \pm 0.05$ & Palomar 60 inch \\
\hline 55354.2 & $i$ & $19.31 \pm 0.03$ & Palomar 60 inch \\
\hline 55356.3 & $i$ & $19.23 \pm 0.16$ & Palomar 60 inch \\
\hline 55363.2 & $i$ & $19.40 \pm 0.05$ & Palomar 60 inch \\
\hline 55368.3 & $i$ & $19.41 \pm 0.05$ & Palomar 60 inch \\
\hline 55372.2 & $i$ & $19.43 \pm 0.07$ & Palomar 60 inch \\
\hline 55381.2 & $i$ & $19.55 \pm 0.06$ & Palomar 60 inch \\
\hline 55391.2 & $i$ & $19.64 \pm 0.06$ & Palomar 60 inch \\
\hline 55406.2 & $i$ & $20.38 \pm 0.13$ & Palomar 60 inch \\
\hline 55307.2 & $J$ & $18.14 \pm 0.29$ & PAIRITEL \\
\hline 55315.2 & $J$ & $18.37 \pm 0.39$ & PAIRITEL \\
\hline 55317.2 & $J$ & $17.89 \pm 0.30$ & PAIRITEL \\
\hline 55319.2 & $J$ & $17.86 \pm 0.26$ & PAIRITEL \\
\hline 55321.2 & $J$ & $17.94 \pm 0.24$ & PAIRITEL \\
\hline 55322.2 & $J$ & $18.38 \pm 0.25$ & PAIRITEL \\
\hline 55324.2 & $J$ & $17.88 \pm 0.21$ & PAIRITEL \\
\hline 55325.2 & $J$ & $17.55 \pm 0.32$ & PAIRITEL \\
\hline 55327.2 & $J$ & $17.86 \pm 0.25$ & PAIRITEL \\
\hline 55331.2 & $J$ & $17.25 \pm 0.18$ & PAIRITEL \\
\hline 55333.2 & $J$ & $17.82 \pm 0.24$ & PAIRITEL \\
\hline 55369.2 & $J$ & $17.78 \pm 0.31$ & PAIRITEL \\
\hline 55307.2 & $H$ & $17.35 \pm 0.21$ & PAIRITEL \\
\hline 55315.2 & $H$ & $17.37 \pm 0.27$ & PAIRITEL \\
\hline 55317.2 & $H$ & $17.14 \pm 0.22$ & PAIRITEL \\
\hline 55319.2 & $H$ & $16.81 \pm 0.27$ & PAIRITEL \\
\hline 55321.2 & $H$ & $17.75 \pm 0.18$ & PAIRITEL \\
\hline 55322.2 & $H$ & $17.25 \pm 0.16$ & PAIRITEL \\
\hline
\end{tabular}


Table 2

(Continued)

\begin{tabular}{lccc}
\hline \hline Date (MJD) & Filter & Mag & Facility \\
\hline 55324.2 & $H$ & $17.22 \pm 0.20$ & PAIRITEL \\
55325.2 & $H$ & $17.19 \pm 0.30$ & PAIRITEL \\
55327.2 & $H$ & $17.02 \pm 0.20$ & PAIRITEL \\
55331.2 & $H$ & $16.97 \pm 0.32$ & PAIRITEL \\
55333.2 & $H$ & $17.07 \pm 0.29$ & PAIRITEL \\
55369.2 & $H$ & $17.22 \pm 0.22$ & PAIRITEL \\
55307.2 & $K$ & $16.17 \pm 0.18$ & PAIRITEL \\
55315.2 & $K$ & $16.56 \pm 0.31$ & PAIRITEL \\
55317.2 & $K$ & $16.84 \pm 0.19$ & PAIRITEL \\
55319.2 & $K$ & $16.90 \pm 0.25$ & PAIRITEL \\
55321.2 & $K$ & $16.84 \pm 0.40$ & PAIRITEL \\
55322.2 & $K$ & $16.69 \pm 0.21$ & PAIRITEL \\
55324.2 & $K$ & $16.29 \pm 0.15$ & PAIRITEL \\
55325.2 & $K$ & $16.73 \pm 0.18$ & PAIRITEL \\
55327.2 & $K$ & $16.65 \pm 0.22$ & PAIRITEL \\
55331.2 & $K$ & $>15.80$ & PAIRITEL \\
55333.2 & $K$ & $>16.60$ & PAIRITEL \\
55369.2 & $K$ & $>16.36$ & PAIRITEL \\
\hline
\end{tabular}

10 minutes by switching between the target field and the point source J1239+0730. The visibility data were calibrated and imaged in the AIPS package following standard practice.

$A$ radio point source was not detected at the position of the transient (Figure 5). After removing extended emission from the host galaxy, the $3 \sigma$ limits for a point source are $93 \mu \mathrm{Jy}$ and $63 \mu \mathrm{Jy}$ at $4.96 \mathrm{GHz}$ and $8.46 \mathrm{GHz}$, respectively (Table 3). At the distance of M99, this corresponds to $L_{v}<2.1 \times 10^{25} \mathrm{erg}$ $\mathrm{s}^{-1} \mathrm{~Hz}^{-1}$. Comparing with the compilation in Chevalier et al. (2006), this upper limit is at the level of the faintest Type II-P (SN 2004dj; Beswick et al. 2005) and Type Ic (SN 2002ap; Berger et al. 2002) supernovae. As noted by Berger et al. (2009), the nearby NGC 300-OT was also not detected in the radio to deeper luminosity limits.

\subsection{Ultraviolet Observations}

We observed PTF 10fqs with Galaxy Evolution Explorer (GALEX; Martin et al. 2005) on two consecutive orbits starting at 2010 April 24.387 (total exposure of 2846 s). All images were reduced and co-added using the standard GALEX pipeline and calibration (Morrissey et al. 2007).

To create a reference image, we co-added 22 images of M99 prior to 2005 April 2 (total exposure of 18571 s). Next, we subtracted the reference image from observations of PTF 10fqs (see Figure 6). No source is detected (Table 3). We find a $3 \sigma$ upper limit of NUV 22.7 AB mag in an aperture consistent with a GALEX point source $\left(7^{\prime \prime} .5 \times 7^{\prime \prime} .5\right)$.

To constrain the pre-explosion counterpart, we measured the limiting magnitude at the position of PTF 10fqs in the co-added reference image. The faintest detected object consistent with being a point source within the galaxy had NUV $=20.1 \mathrm{AB}$ mag. The $3 \sigma$ limit based on measuring the sky rms is NUV > 21.8 AB mag.

\subsection{X-ray Observations}

We observed PTF 10fqs with Swift/XRT on April 20.466 for $2507.3 \mathrm{~s}$ and April 22.024 for $2623.5 \mathrm{~s}$. No source is detected to a $3 \sigma$ limiting count rate (assuming an $18^{\prime \prime}$ radius) of $4.6 \times 10^{-4}$ counts $\mathrm{s}^{-1}$. Assuming a power-law model with a photon index of two, this corresponds to a flux limit of $1.6 \times 10^{-14} \mathrm{erg} \mathrm{cm}^{-2} \mathrm{~s}^{-1}$.

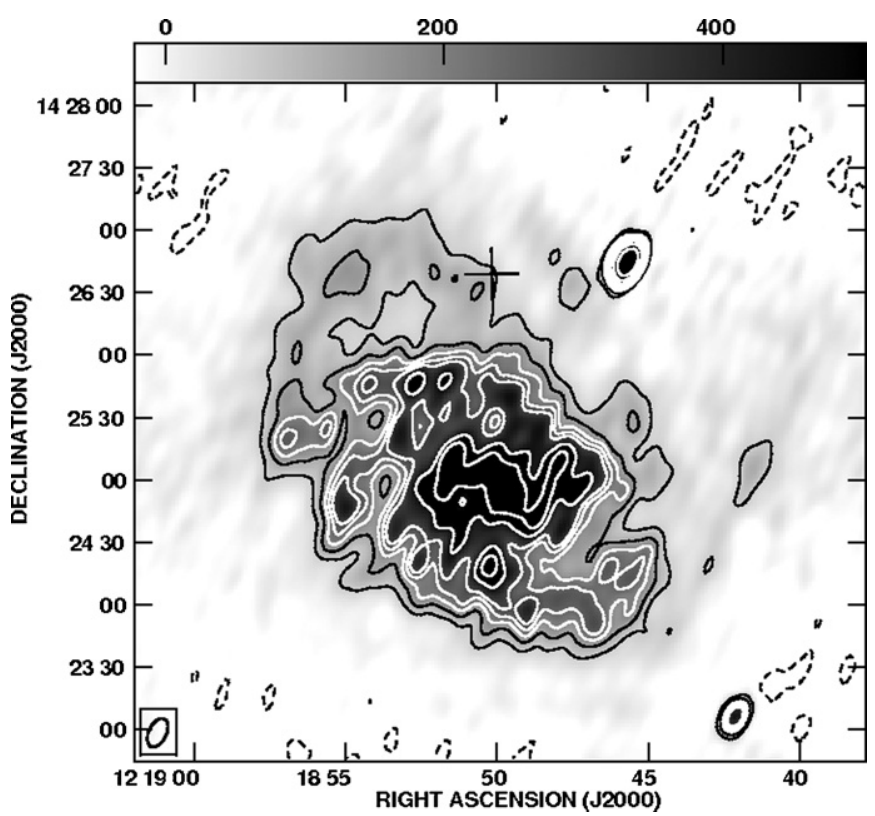

Figure 5. Observation of PTF 10fqs (denoted by a plus sign) with the EVLA at $4.96 \mathrm{GHz}$, just four days after discovery. The gray-scale range is -40 to $1000 \mu \mathrm{Jy}$ per beam and the size of the synthesized beam is shown at the bottom left corner.

\section{ARCHIVAL DATA}

\subsection{Hubble Space Telescope (HST)}

A query to the Hubble Legacy archive returned HST images of M99 in the F606W (2001), F336W (2009), and F814W (2009) filters. We multidrizzled these data (PI: Regan; Proposal ID 11966) and registered our Gemini/GMOS acquisition image with the HST/WFPC2 images. Unfortunately, PTF 10fqs is just off the edge of the chip for the F606W filter image.

The total $1 \sigma$ registration error, added in quadrature, was 0.59 pixels. Their sources of error are as follows: centroiding error ( 0.17 in $x, 0.30$ in $y)$, registration error between the Gemini image and the HST/F814W image (0.19 in $x, 0.44$ in $y$ ), and registration error between the $H S T / \mathrm{F} 814 \mathrm{~W}$ image and the $H S T /$ F336W image (0.04 in $x, 0.02$ in $y$ ). Hence, in Figure 7, we plot a $5 \sigma$ radius of 3 pixels or 0.27 .

No source is detected at the location of PTF 10fqs. To estimate the limiting magnitude, we ran SExtractor and performed photometry following Holtzman et al. (1995). We find $3 \sigma$ limiting Vega magnitudes of $I>26.9$ and $U>26$ in the $1800 \mathrm{~s}$ and $6600 \mathrm{~s}$ exposures, respectively.

\subsection{Spitzer Space Telescope}

M99 was part of the sample of the SIRTF Nearby Galaxies Survey (SINGS) galaxies (Kennicutt et al. 2003). This program undertook IRAC and MIPS imaging in 2004-2005. No point source is detected at the location of PTF 10fqs (see Figure 8). We downloaded IRAC images from the final data release of SINGS and MIPS images from the standard Spitzer pipeline. Computed upper limits (see Table 4) assume a 2 pixel aperture radius and sky rms based on a $20 \times 20$ pixel box at the location.

\subsection{Katzman Automatic Imaging Telescope}

The $0.76 \mathrm{~m}$ Katzman Automatic Imaging Telescope (KAIT ${ }^{22}$; Li et al. 2000; Filippenko et al. 2001) had extensively imaged

\footnotetext{
22 http://astro.berkeley.edu/ bait/kait.html.
} 


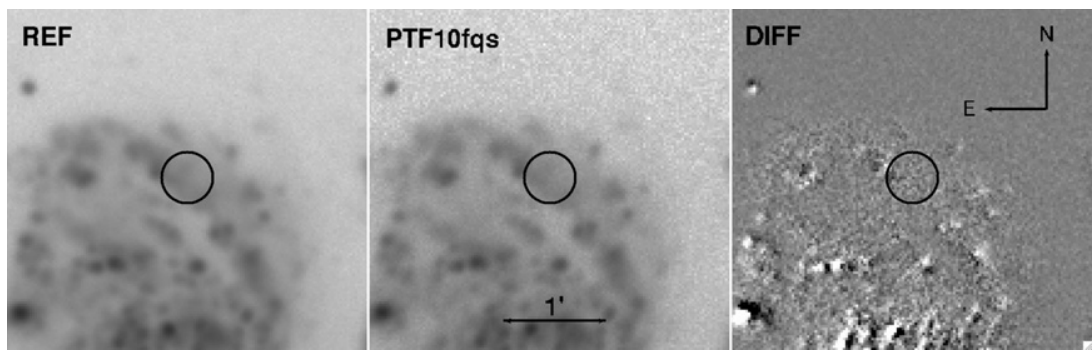

Figure 6. Observation of PTF 10fqs with GALEX. Reference data are taken from 22 images between 2005 March 28 and 2005 April 2 (left panel). Observations of PTF 10fqs were taken on 2010 April 24 (center panel). No source is detected in the difference image (right panel).
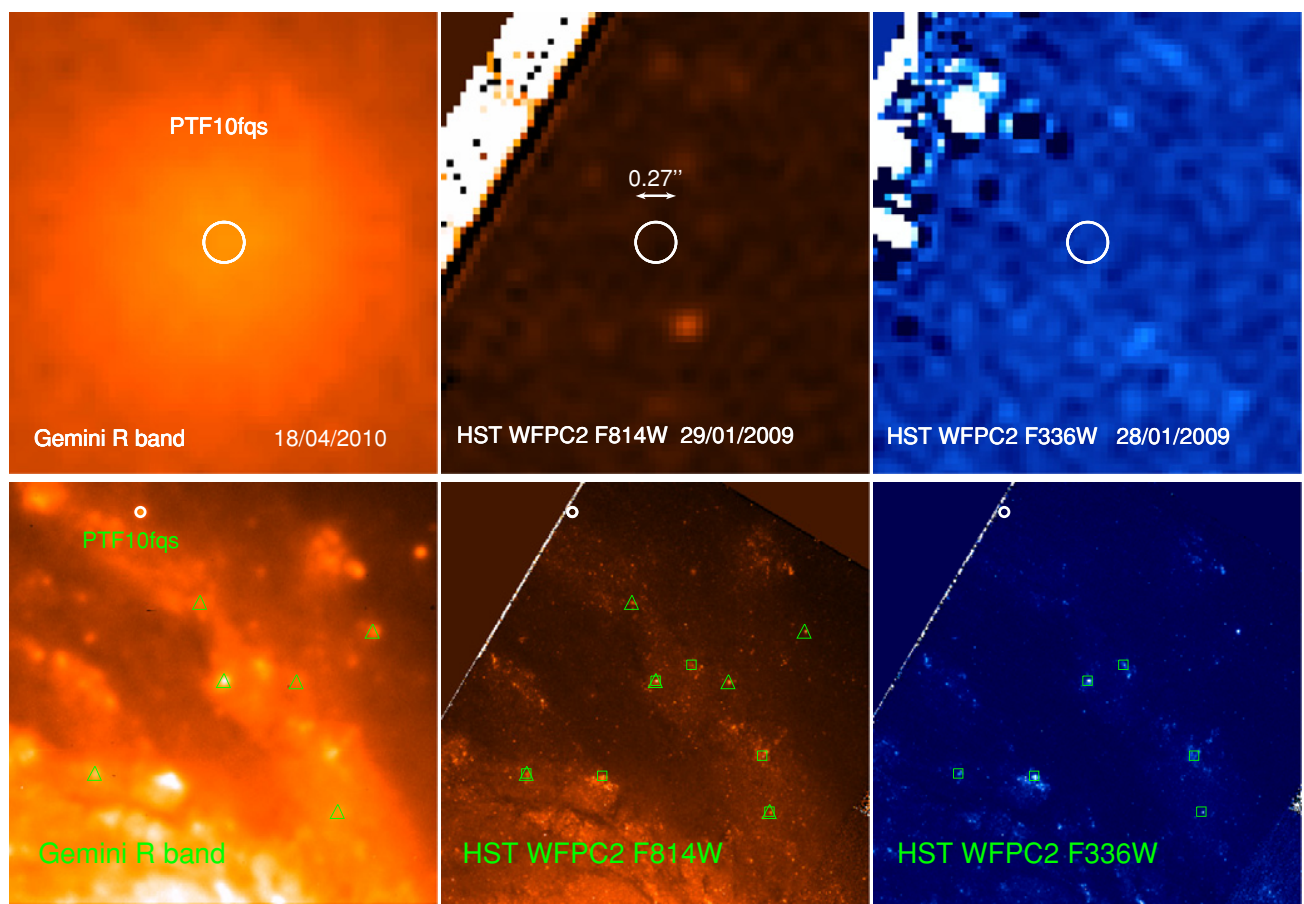

Figure 7. $H S T / \mathrm{F} 814 \mathrm{~W}$ and $H S T / \mathrm{F} 336 \mathrm{~W}$ observations from 2009. Top panel: zoomed-in view $\left(22^{\prime \prime} 8 \times 22^{\prime \prime} 6\right)$ to show the absence of a pre-explosion counterpart. This rules out red supergiants fainter than $M_{V}=-3 \mathrm{mag}$ and blue supergiants fainter than $M_{V}=-4.3 \mathrm{mag}$. Bottom panel: zoomed-out view (81'.2 $\times 82$ ". 1$)$ to show registration stars. Stars used to register the Gemini/R-band image with the HST/F814W image are denoted by triangles. Stars used to register the $H S T / F 814 \mathrm{~W}$ image with the $H S T / \mathrm{F} 336 \mathrm{~W}$ are denoted by squares.

(A color version of this figure is available in the online journal.)

Table 3

PTF 10fqs Broadband Measurements

\begin{tabular}{llllll}
\hline \hline $\begin{array}{c}\text { Date } \\
\text { (UT 2010) }\end{array}$ & MJD & Filter & Magnitude/Flux & $\begin{array}{c}v \\
(\mathrm{~Hz})\end{array}$ & $\begin{array}{c}v F_{v} \\
\left(\mathrm{erg} \mathrm{cm}^{-2} \mathrm{~s}^{-1}\right)\end{array}$ \\
\hline Apr 20.23 & 55306.23 & $4.96 \mathrm{GHz}$ & $<93 \mu \mathrm{Jy}$ & $4.960 \times 10^{9}$ & $4.613 \times 10^{-18}$ \\
Apr 20.23 & 55306.23 & $8.46 \mathrm{GHz}$ & $<63 \mu \mathrm{Jy}$ & $8.460 \times 10^{9}$ & $5.330 \times 10^{-18}$ \\
Apr 20.466 & 55306.466 & $0.3-10 \mathrm{keV}$ & $<4.6 \times 10^{-4} \mathrm{cps}$ & $4.200 \times 10^{17}$ & $2.864 \times 10^{-15}$ \\
Apr 24.646 & 55310.646 & NUV (AB) & $>22.7 \mathrm{mag}$ & $1.295 \times 10^{15}$ & $3.885 \times 10^{-14}$ \\
\hline
\end{tabular}

M99 in the past decade-113 images in the period 1999-2010. We stacked the images in each season and find no point source at the location of PTF 10fqs. Limiting magnitudes for each season are summarized in Table 5.

\subsection{DeepSky Imaging}

DeepSky $^{23}$ (Nugent 2009) also has imaging at the position of this field over the interval 2006-2008. No point source is detected in a yearly sum of these images (see Table 5).

\footnotetext{
23 http://supernova.lbl.gov/ nugent/deepsky.html.
}

\section{ANALYSIS}

\subsection{SED}

We fit a blackbody spectrum to the optical and near-infrared fluxes of PTF 10fqs without taking into account any local extinction. The best fit gives a lower limit on the temperature of $\sim 3900 \mathrm{~K}$

\subsection{Spectral Modeling}

We combined the four spectra obtained with HET (between +5 days and +17 days). The most prominent (narrow) features in 
Table 4

Progenitor Constraints for PTF 10fqs

\begin{tabular}{llll}
\hline \hline Date & \multicolumn{1}{c}{ Filter } & Magnitude/Flux & \multicolumn{1}{c}{ Facility } \\
\hline 2005 & NUV $(\mathrm{AB})$ & $>21.8 \mathrm{mag}$ & GALEX \\
2009 & F336W (Vega $U)$ & $>26 \mathrm{mag}$ & $H S T /$ WFPC2 \\
2009 & F814W (Vega $I)$ & $>26.9 \mathrm{mag}$ & HST/WFPC2 \\
2004 & $3.6 \mu \mathrm{m}$ & $<5.3 \mu \mathrm{Jy}$ & Spitzer $/$ IRAC \\
2004 & $4.5 \mu \mathrm{m}$ & $<3.5 \mu \mathrm{Jy}$ & Spitzer $/$ IRAC \\
2004 & $5.8 \mu \mathrm{m}$ & $<51 \mu \mathrm{Jy}$ & Spitzer $/$ IRAC \\
2004 & $8.0 \mu \mathrm{m}$ & $<344 \mu \mathrm{Jy}$ & Spitzer $/$ IRAC \\
2004 & $23.68 \mu \mathrm{m}$ & $<240 \mu \mathrm{Jy}$ & Spitzer $/$ MIPS \\
\hline
\end{tabular}

Table 5

Historical Optical Observations

\begin{tabular}{lccc}
\hline \hline \multicolumn{1}{c}{$\begin{array}{c}\text { Date Range } \\
(\mathrm{UT})\end{array}$} & $\begin{array}{c}\text { Exposure } \\
(\mathrm{s})\end{array}$ & $\begin{array}{c}\text { Limiting Mag } \\
(R \text { Band })\end{array}$ & Facility \\
\hline 1998 Dec 27-1999 Jun 1 & 680.0 & $>20.4$ & KAIT \\
1999 Nov 26-2000 Jun 7 & 567.0 & $>20.4$ & KAIT \\
2001 Apr 11-2001 Jun 7 & 192.0 & $>20.1$ & KAIT \\
2002 Jan 14-2002 Jun 8 & 486.0 & $>20.4$ & KAIT \\
2003 Jan 15-2003 Jun 4 & 318.0 & $>20.4$ & KAIT \\
2004 Jan 29-2004 Jun 16 & 392.0 & $>20.3$ & KAIT \\
2004 Dec 25-2005 Jun 1 & 110.0 & $>20.3$ & KAIT \\
2006 Jan 12-2006 May 18 & 665.7 & $>22.2$ & DeepSky \\
2006 Mar 24-2006 May 18 & 78.0 & $>20.4$ & KAIT \\
2007 Jan 4-2007 May 6 & 1749.9 & $>22.4$ & DeepSky \\
2007 Jan 13-2007 Jun 4 & 178.0 & $>20.4$ & KAIT \\
2007 Dec 22-2008 Jun 16 & 332.0 & $>20.4$ & KAIT \\
2008 May 18-2008 May 18 & 241.2 & $>20.7$ & DeepSky \\
2009 Mar 28-2009 Apr 27 & 64.0 & $>20.3$ & KAIT \\
2010 Feb 11-2010 Mar 22 & 32.0 & $>20.0$ & KAIT \\
\hline
\end{tabular}

Note. All images in a season were stacked.

the spectra of PTF $10 \mathrm{fqs}$ are $\mathrm{H} \alpha$, [Ca II], the Ca II near-IR triplet, $\mathrm{Na}$ I D, and $\mathrm{H} \beta$. The measured line fluxes and equivalent widths are summarized in Table 6 . The $\mathrm{H} \alpha$ FWHM is $\approx 930 \mathrm{~km} \mathrm{~s}^{-1}$ (taking into account the instrumental resolution).

The Ca II near-IR triplet is of particular interest. The HET spectra appear to show a flux excess longward of $8300 \AA$ A beyond that expected from a simple, low-order polynomial fit to the continuum. Together with a possible broad flux deficit near $8300 \AA$, the overall effect suggests a P-Cygni profile. If we fit three Gaussians, the Ca II near-IR triplet features are broader than the [Ca II] doublet, and quite likely even broader than the narrow component of the $\mathrm{H} \alpha$ profile. There is a surplus of flux at $8600 \AA$, which falls right between the 8498.02, 8542.09 $\AA$ pair and the more isolated $8662.14 \AA$ line, such as one would expect from an underlying broad feature.

We test this hypothesis further with SYNOW (Jeffery \& Branch 1990) modeling. We do not get a good fit to the overall shape of the spectrum with an extinguished blackbody of any temperature (assuming standard dust). To fit the red end of the spectrum, we need high temperature and extinction (consistent with the strong Na I D absorption). We find that in addition to narrow emission from $\mathrm{Ca}$ II IR, there is also a likely underlying broad component (see Figure 9). The width (FWHM) of this feature is $\approx 10,000 \mathrm{~km} \mathrm{~s}^{-1}$.

A caveat to this interpretation is that a similar broad feature is not seen in the $\mathrm{H} \alpha$ profile. However, as noted below (Section 6.2), reinspection of the spectra of related transients shows possible evidence of a similar broad feature. Thus, we cautiously accept the interpretation that in addition to the low-

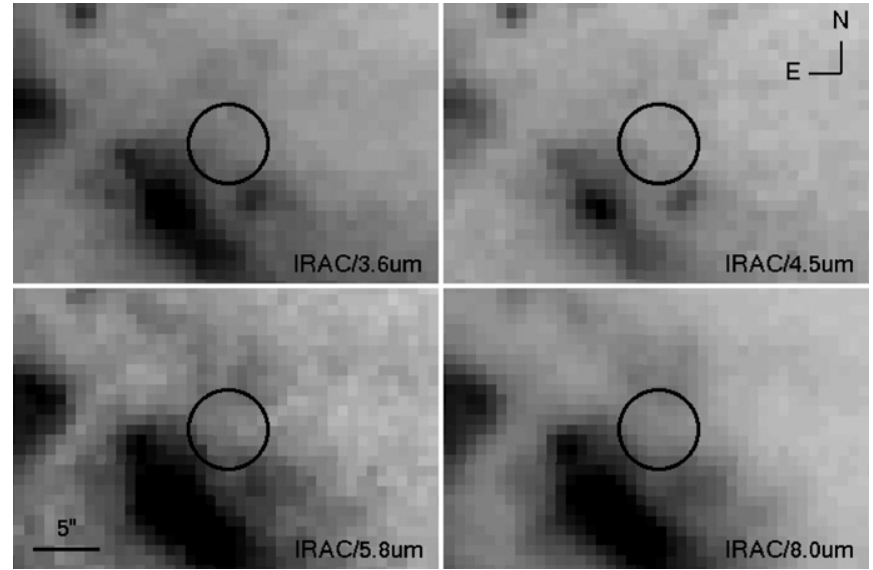

Figure 8. Pre-explosion observations with Spitzer/IRAC. No source is found to be consistent with PTF $10 \mathrm{fqs}$.

Table 6

PTF 10fqs Spectrum Properties

\begin{tabular}{lcrr}
\hline \hline Line & $\begin{array}{c}\text { Obs } \lambda \\
(\AA)\end{array}$ & $\begin{array}{c}\text { Flux } \\
\left(\mathrm{erg} \mathrm{cm}^{-2} \mathrm{~s}^{-1}\right)\end{array}$ & $\begin{array}{c}\text { Equivalent Width } \\
(\AA)\end{array}$ \\
\hline $\mathrm{H} \alpha$ & 6621.2 & $1.0 \times 10^{-15}$ & -19.9 \\
$\mathrm{H} \beta$ & 4907.3 & $1.3 \times 10^{-16}$ & -3.7 \\
$\mathrm{Na}$ I D & 5939.0 & $-3.1 \times 10^{-16}$ & 6.4 \\
{$[\mathrm{Ca}$ II $]$} & 7355.8 & $2.9 \times 10^{-16}$ & -6.1 \\
{$[\mathrm{Ca}$ II $]$} & 7387.2 & $1.8 \times 10^{-16}$ & -3.7
\end{tabular}

Note. Above line fluxes are measured on combined HET spectra (phase between +5 days and +17 days).

velocity outflow seen in $\mathrm{H} \alpha$, there is a higher velocity outflow in this explosion.

\section{WHAT IS PTF 10fqs?}

In a nutshell, PTF 10fqs is a red transient with a peak luminosity of $M_{r}=-12.3$ and a spectrum dominated by $\mathrm{H} \alpha$, [Ca II], and CaII emission. The width of the $\mathrm{H} \alpha$ line is $\approx 930 \mathrm{~km} \mathrm{~s}^{-1}$, and there is some evidence for $\mathrm{a} \approx 10,000 \mathrm{~km} \mathrm{~s}^{-1}$ broad Ca II IR feature.

The peak absolute magnitude and the $\mathrm{H} \alpha$ line width of PTF 10fqs are similar to those seen in M85OT2006-1 (hereafter M85-OT; Kulkarni et al. 2007), SN 2008S (Prieto et al. 2008; Smith et al. 2009), and NGC 300-OT (Bond et al. 2009; Berger et al. 2009). However, there are some differences amongst these four sources. Thus, to aid a better classification, we review the similarities and differences between these four sources.

\subsection{The Light Curve}

The light curves of all four transients (PTF 10fqs, SN 2008S, NGC 300-OT, and M85-OT) were red and evolved slowly for the first couple of months. PTF 10fqs had a well-sampled rise (Figure 4) - it rose by $1.1 \mathrm{mag}$ in the $r$ band in 10.8 days. After maximum, PTF 10fqs declined slowly in the $r$ band by 1 mag in 68 days. Subsequently, it evolved more rapidly, declining by the next $1.3 \mathrm{mag}$ in 16 days. PTF $10 \mathrm{fqs}$ had $g-r=1.0$ at peak and declined relatively faster in the $g$ band (1 mag in 40 days) than the $r$-band. In comparison, SN $2008 \mathrm{~S}$ declined by $1 \mathrm{mag}$ in 51 days in the $R$ band and 44 days in the $V$ band. The epoch of maximum light is uncertain for NGC 300-OT due to lack of observations and is constrained to be anywhere between 2008 April 24 and May 15 (Bond et al. 2009). If we assume it to be 


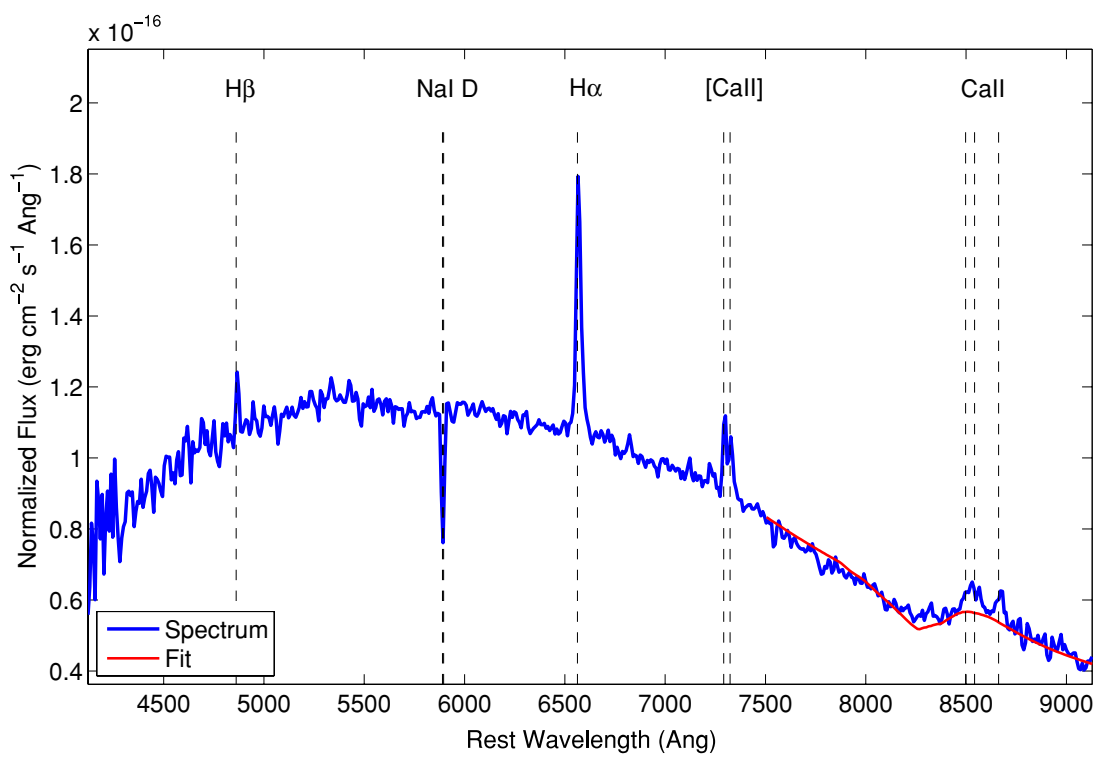

Figure 9. SYNOW fit to summed HET spectra of PTF 10fqs. Note the broad, possibly P Cygni, feature under the Ca II near-IR triplet.

(A color version of this figure is available in the online journal.)

April 27, the evolution is that $R$ band and $I$ band are similar to that for PTF 10fqs (Figure 4).

\subsection{The Spectrum}

The spectral evolution of SN 2008S (Botticella et al. 2009) and NGC 300-OT (Berger et al. 2009) were very well studied as they were in very nearby galaxies. We took this opportunity to reanalyze the spectrum of M85-OT reported by Kulkarni et al. $(2007)^{24}$.

Armed thus, we compare and contrast the spectral features of these four transients (see Figure 10).

1. The $\mathrm{H} \alpha$ profile of $\mathrm{SN} 2008 \mathrm{~S}$ showed a narrow component (unshocked circumstellar material $\left(\mathrm{CSM} ; \approx 250 \mathrm{~km} \mathrm{~s}^{-1}\right.$ ), an intermediate component (shocked material between the ejecta and the CSM; $\approx 1000 \mathrm{~km} \mathrm{~s}^{-1}$ ), and a broad component (underlying ejecta emission; $\approx 3000 \mathrm{~km} \mathrm{~s}^{-1}$ ). NGC 300-OT exhibited narrow (560 $\left.\mathrm{km} \mathrm{s}^{-1}\right)$ and intermediate-width components $\left(1100 \mathrm{~km} \mathrm{~s}^{-1}\right)$. M85-OT only had a narrow component $\left(350 \mathrm{~km} \mathrm{~s}^{-1}\right)$. PTF $10 \mathrm{fqs}$ shows an intermediate-width component $\left(930 \mathrm{~km} \mathrm{~s}^{-1}\right)$ in the $\mathrm{H} \alpha$ emission line.

2. SN 2008S had an $\mathrm{H} \alpha / \mathrm{H} \beta$ ratio that evolved from 4 to 10. NGC 300-OT had a ratio of 6, while M85-OT showed a ratio of 3.5 . PTF $10 \mathrm{fqs}$ has a ratio of 6.5 . All events show flux ratios higher than 3.1 (the expectation from Case B recombination). This may be evidence for collisional excitation (Drake \& Ulrich 1980).

3. PTF 10fqs, NGC 300-OT, and SN 2008S exhibit three calcium features: $\mathrm{Ca}$ II $\mathrm{H} \& \mathrm{~K}$ in absorption, [Ca II] and $\mathrm{Ca}$ II near-IR triplet in emission. A reanalysis of M85-OT shows $\mathrm{Ca}$ II H\&K , as well as lower signal-to-noise ratio detections of both [Ca II] and Ca II IR. Smith et al. (2009) show a similarity between the spectra of SN2008S and a Galactic

\footnotetext{
24 In addition to the features mentioned by Kulkarni et al. (2007), we can securely identify Ca II H\&K and see evidence of [Ca II] and the Ca II near-IR triplet. Furthermore, we can identify the lines previously marked

"unidentified": $4115 \AA$ is $\mathrm{H} \gamma, 6428 \AA$ is likely Fe II (multiplet 74), $6527 \AA$ is likely Fe II (multiplets 40 and 92).
}

hypergiant (IRC+10420) and suggest that strong [Ca II] is due to destruction of dust grains.

4. As noted earlier (see also Figure 9), there is evidence for a broad feature around $8600 \AA$ in the spectrum of PTF 10 fqs. Motivated by this finding, we reinspected the spectra of previous transients and find that a similar broad feature may also be present in the spectra of M85-OT and NGC 300-OT.

5. Narrow Fe II lines are visible in NGC 300-OT and SN 2008S. Reanalysis of M85-OT spectra possibly shows Fe II (74) and Fe II (40, 92).

6. For SN 2008S, Na I D evolves from strong absorption at early times to emission at very late times. This suggests a very dense CSM. O I $\lambda 7774$ is also in emission at late times. For NGC 300-OT, Na I D has a much lower equivalent width at early times, but it also evolves from absorption to emission. Neither Na I D nor O I are seen in M85-OT, but there is possibly $\mathrm{K}_{\mathrm{I}}$ in emission. PTF $10 \mathrm{fqs}$ has an equivalent width of $\mathrm{Na}$ I D of 6.4, higher than SN 2008S (2.3-4.4) and NGC 300-OT (1.0-2.1). The equivalent width of $\mathrm{Na}$ I $\mathrm{D}$ is too high to apply a standard correlation to estimate extinction.

\subsection{The Pre-explosion Counterpart}

We plot the upper limits on the pre-explosion counterpart for PTF 10fqs in Figure 11. The most constraining limits are in the optical. Following the Geneva stellar evolution tracks (Lejeune \& Schaerer 2001) for unenshrouded stars, the luminosity limit of $M_{I}>-4.3$ corresponds to a progenitor mass $<4 M_{\odot}$. If there was extinction of, say $1.5 \mathrm{mag}$, this would change the limit to $<7 M_{\odot}$. None of SN 2008S, NGC 300-OT, M85-OT, and PTF 10fqs have an optical counterpart in deep, pre-explosion optical images. The limits in all cases are deep enough to at least rule out red supergiants and blue supergiants.

For both SN 2008S and NGC 300-OT, an extremely red and luminous mid-infrared pre-explosion counterpart is seen (Prieto et al. 2008; Thompson et al. 2009). Recently, Khan et al. (2010) show that such progenitors are as rare as one per galaxy (and possibly associated with a very short-lived phase of many massive stars). Thus, both of these transients can be reasonably 

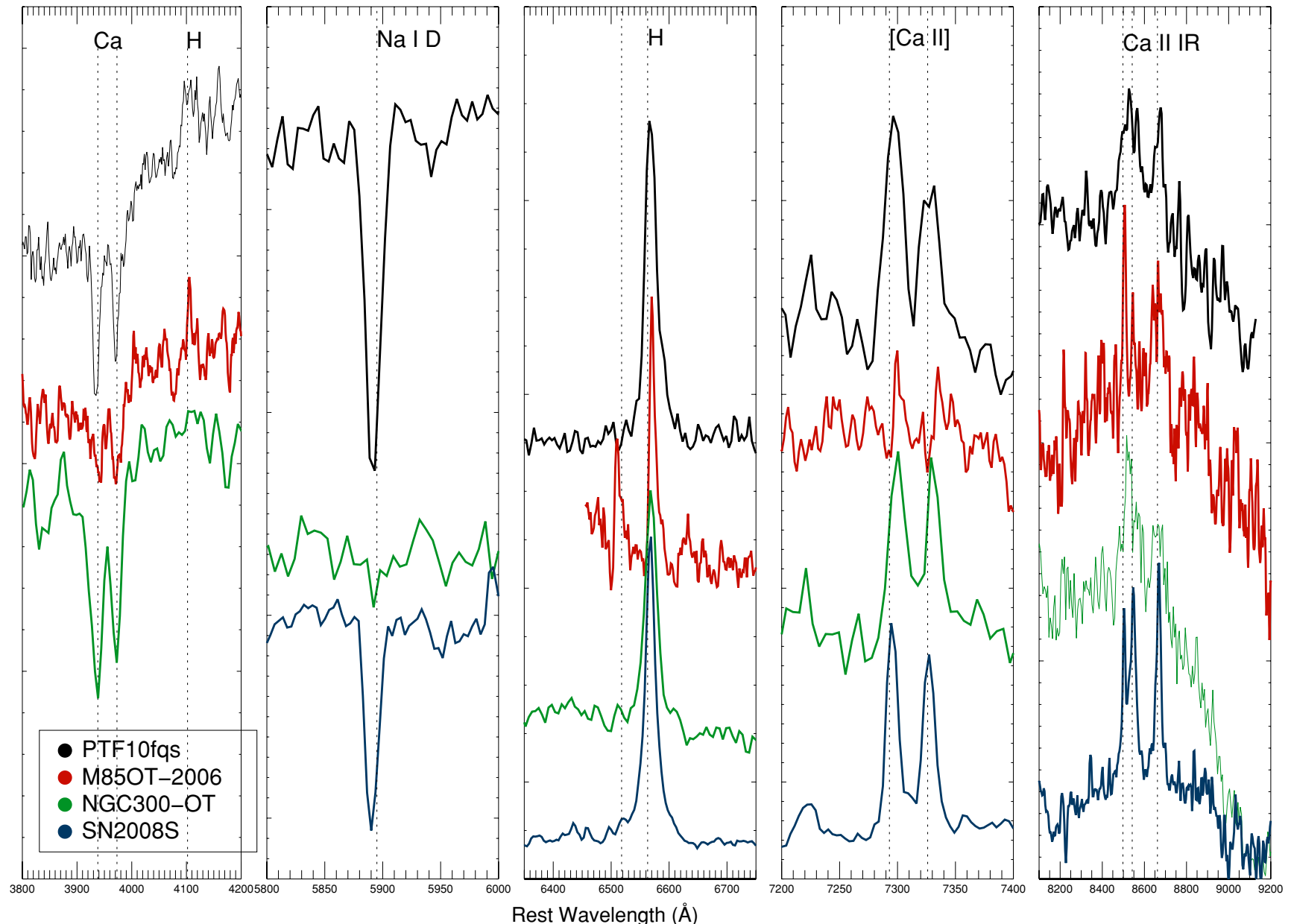

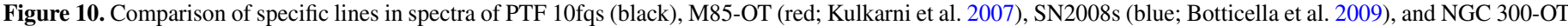

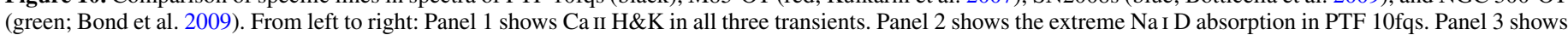

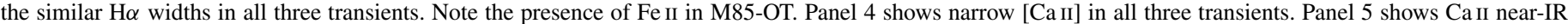
triplet. Note that in addition to the narrow lines, there is possibly an underlying broad feature.

(A color version of this figure is available in the online journal.)

associated with massive stars. Unfortunately, the large distance to M85 and M99 means that the pre-explosion Spitzer limits on M85-OT and PTF 10fqs are not deep enough by a factor of few to constrain their progenitors to similar depths (see Figure 12).

\subsection{The Large-scale Environment}

M85-OT is located in the lenticular galaxy M85 (also in the Virgo Cluster). Fortunately, this galaxy was observed with HST for the ACS Virgo Cluster Survey as well as for a GO program. The transient is not associated with any star-forming region and the absolute magnitude of the progenitor is fainter than $M_{g} \approx-4\left(<7 M_{\odot}\right.$ not correcting for extinction; Ofek et al. 2008). Thus, a massive-star origin is quite unlikely.

In contrast, SN 2008S, NGC 300-OT, and PTF 10fqs occurred in star-forming galaxies. It may be worth noting here that three supernovae (all of the core-collapse variety) have previously been discovered in the host galaxy of PTF 10fqs. It is perhaps of some significance that eight supernovae (six core collapse, two unclassified) were discovered in NGC 6946 in addition to SN 2008S. Only one supernova (of Type Ia) has been discovered in NGC 300. Small-number statistics and discovery bias (incompleteness from variety of different searches) notwithstanding, we make the suggestion that galaxies with a high supernova rate preferentially produce luminous red novae.

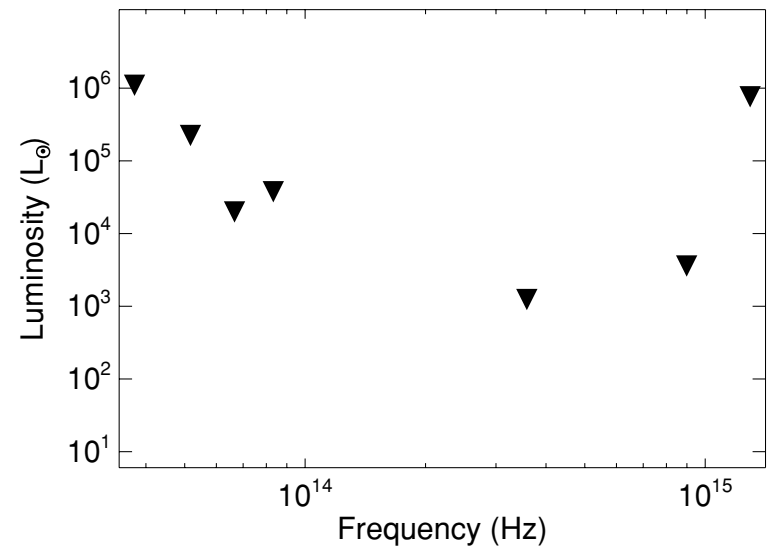

Figure 11. Spectral energy distribution (mid-IR to UV) constraints on the preexplosion counterpart of PTF 10fqs. Upper limits are denoted by downward arrows.

If this suggestion is correct, then it would be worth the effort to systematically maintain close vigilance on the nearest galaxies having large supernova rates.

Kulkarni et al. (2007) suggested that V838 Mon, V4332 Sgr, and M31 RV may also be luminous, red novae. We note here that the two Galactic sources are located in star-forming regions. 


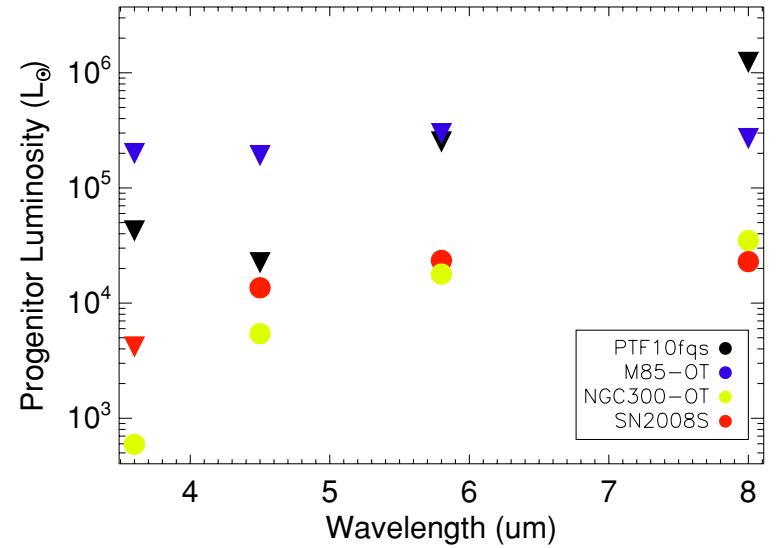

Figure 12. Pre-explosion detections (circles) or upper limits (downward triangles) from Spitzer for PTF 10fqs, SN 2008S, NGC 300-OT, and M85OT. The non-detection of a progenitor for PTF 10fqs and M85-OT does not rule out a progenitor of luminosity comparable to that detected for NGC 300-OT and SN 2008S.

(A color version of this figure is available in the online journal.)

Specifically, V838 Mon is in a young (25 Myr) star cluster and may even have a B3 companion (Afşar \& Bond 2007). V4332 Sgr (Martini et al. 1999) is located toward the inner Galaxy (in Sagittarius). On the other hand, M31 RV is located in the bulge of M31. HST observations (undertaken with WFPC2 in parallel mode) taken about a decade ago show that the immediate environs of M31-RV are typical bulge-population stars (Bond \& Siegel 2006). No unusual remnant star is seen at the astrometric position of M31 RV, nor any evidence of a light echo (consistent with the absence of dense circumstellar or interstellar gas that is essential to form echoes). Separately, there is no evidence for any luminous outbursts in this area in the period 1942-1993 (Boschi \& Munari 2004). Thus, M31 RV appears to have been a cataclysmic event in the bulge of M31.

\section{CONCLUSION}

PTF 10fqs is the fourth member of a class of extragalactic transients $^{25}$ which possess a peak luminosity between that of novae and supernovae, and have spectral and photometric evolution that bear no resemblance to either supernovae or novae. The other members of this class are M85-OT, NGC 300OT, and SN 2008S.

NGC 300-OT and SN 2008S are remarkable for their very bright mid-infrared progenitors. Though sensitive pre-explosion observations of M85-OT and PTF 10fqs do exist, the large distance to the Virgo Cluster $(17 \mathrm{Mpc})$ relative to that of NGC 300 (1.9 Mpc) and NGC 6946 (5.7 Mpc) results in weak constraints on the luminosity of any pre-explosion star. PTF 10fqs, NGC 300-OT, and SN 2008S occurred in starforming regions whereas M85-OT was in the bulge. Prima facie, this group of explosive events can be divided into a disk and a bulge group.

The discovery of PTF 10fqs in itself cannot address whether the two groups of luminous, red novae are one and the same. The proposed models to explain this group are diverse: electron capture within an extreme asymptotic giant branch (AGB) star, common-envelope phase (stellar merger), inspiral of a giant planet into the envelope of an aging parent star, a most peculiar nova, and a most peculiar supernova.

\footnotetext{
25 Henceforth we use the term "luminous red novae" as a functional short name for such events.
}

The possible evidence of the broad feature centered around the Ca II near-IR triplet with an inferred velocity spread of $10,000 \mathrm{~km} \mathrm{~s}^{-1}$ may be an important clue. It would mean that these events possess both a low- and a high-velocity outflow. By comparison with other astronomical sources, one can envisage a high-velocity polar outflow and a slower equatorial outflow (but with a larger mass). To this end, continued sensitive spectroscopy of PTF 10fqs (and of course other such future events) would be very valuable.

The "Transients in the Local Universe" key project of the PTF is designed to systematically unveil events in the gap between novae and supernovae. It surveys $\approx 20,000$ nearby galaxies $(d<200 \mathrm{Mpc})$ yearly at 1 day cadence and a depth of $R<21 \mathrm{mag}$. (If the maximum luminosity of this class is $-14 \mathrm{mag}$, then we would be sensitive to events out to $100 \mathrm{Mpc}$.) Furthermore, Spitzer has a growing archive of deep images of nearby galaxies (e.g., SINGS; Kennicutt et al. 2003; LVL, Dale et al. 2009, and S4G, Sheth et al. 2010), and WISE (Wright et al. 2010) has an ongoing all-sky survey in the mid-IR. This will allow us to probe deeper in search of the pre-explosion counterpart and possibly present a new channel for discovery of luminous red novae. The discovery of PTF 10fqs is only the harbinger of the uncovering of a large sample of such transients to unveil the nature of this new class of explosions.

M.M.K. thanks the Gordon and Betty Moore Foundation for a Hale Fellowship in support of graduate study. The Weizmann Institute PTF participation is supported in part by the Israel Science Foundation via grants to A.G.Y. The Weizmann-Caltech collaborative PTF effort is supported by the US-Israel Binational Science Foundation. A.G.Y. and M.S. are jointly supported by the "making connections" Weizmann-UK program. A.G.Y. further acknowledges support by a Marie Curie IRG fellowship and the Peter and Patricia Gruber Award, as well as funding by the Benoziyo Center for Astrophysics and the Yeda-Sela center at the Weizmann Institute. A.V.F.'s group and KAIT are supported by National Science Foundation (NSF) grant AST-0908886, the Sylvia \& Jim Katzman Foundation, the Richard \& Rhoda Goldman Fund, Gary and Cynthia Bengier, and the TABASGO Foundation; additional funding was provided by NASA through Spitzer grant 1322321, as well as HST grant AR-11248 from the Space Telescope Science Institute, which is operated by Associated Universities for Research in Astronomy, Inc., under NASA contract NAS 5-26555. J.S.B. and his group are partially funded by a DOE SciDAC grant. E.O.O. and D.P. are supported by the Einstein fellowship. L.B. is supported by the National Science Foundation under grants PHY 05-51164 and AST 07-07633.

We are grateful to the staff of the Gemini Observatory for their promptness and high efficiency in attending to our TOO request. Likewise, we thank the staff of the Very Large Array and the Hobby-Eberly Telescope. We acknowledge the following internet repositories: SEDS (Messier Objects) and GOLDMine (Virgo Cluster), Finally, as always, we are grateful to the librarians who maintain the ADS, the NED, and SIMBAD data systems.

The Hobby-Eberly Telescope (HET) is a joint project of the University of Texas at Austin, the Pennsylvania State University, Stanford University, Ludwig-Maximillians-Universität München, and Georg-August-Universität Göttingen. The HET is named in honor of its principal benefactors, William P. Hobby and Robert E. Eberly. The Marcario LRS is named for Mike Marcario of High Lonesome Optics, who fabricated several 
optics for the instrument but died before its completion; it is a joint project of the Hobby-Eberly Telescope partnership and the Instituto de Astronomía de la Universidad Nacional Autónoma de México. GALEX (Galaxy Evolution Explorer) is a NASA Small Explorer, launched in 2003 April. We gratefully acknowledge NASA's support for construction, operation, and science analysis for the GALEX mission, developed in cooperation with the Centre National d'Etudes Spatiales of France and the Korean Ministry of Science and Technology. PAIRITEL is operated by the Smithsonian Astrophysical Observatory (SAO) and was made possible by a grant from the Harvard University Milton Fund, the camera loan from the University of Virginia, and the continued support of the SAO and UC Berkeley. The Expanded Very Large Array is operated by the National Radio Astronomy Observatory, a facility of the NSF operated under cooperative agreement by Associated Universities, Inc.

\section{REFERENCES}

Abazajian, K. N., et al. 2009, ApJS, 182, 543

Afşar, M., \& Bond, H. E. 2007, AJ, 133, 387

Barbon, R., Ciatti, F., \& Rosino, L. 1973, A\&A, 29, 57

Berger, E., Kulkarni, S. R., \& Chevalier, R. A. 2002, ApJ, 577, L5

Berger, E., et al. 2009, ApJ, 699, 1850

Beswick, R. J., Muxlow, T. W. B., Argo, M. K., Pedlar, A., Marcaide, J. M., \& Wills, K. A. 2005, ApJ, 623, L21

Bildsten, L., Shen, K. J., Weinberg, N. N., \& Nelemans, G. 2007, ApJ, 662, L95

Bloom, J. S., Starr, D. L., Blake, C. H., Skrutskie, M. F., \& Falco, E. E. 2006, in ASP Conf. Ser. 351, Astronomical Data Analysis Software and Systems XV, ed. C. Gabriel, C. Arviset, D. Ponz, \& S. Enrique (San Francisco, CA: ASP), 751

Bond, H. E., Bedin, L. R., Bonanos, A. Z., Humphreys, R. M., Monard, L. A. G. B., Prieto, J. L., \& Walter, F. M. 2009, ApJ, 695, L154

Bond, H. E., \& Siegel, M. H. 2006, AJ, 131, 984

Boschi, F., \& Munari, U. 2004, A\&A, 418, 869

Botticella, M. T., et al. 2009, MNRAS, 398, 1041

Cenko, S. B., et al. 2006, PASP, 118, 1396

Chevalier, R. A., Fransson, C., \& Nymark, T. K. 2006, ApJ, 641, 1029

Cohen, M., Wheaton, W. A., \& Megeath, S. T. 2003, AJ, 126, 1090

Dale, D. A., et al. 2009, ApJ, 703, 517

Drake, S. A., \& Ulrich, R. K. 1980, ApJS, 42, 351

Fairall, A. P. 1972, Mon. Notes Astron. Soc. South. Afr., 31, 23

Filippenko, A. V., Li, W. D., Treffers, R. R., \& Modjaz, M. 2001, in ASP Conf. Ser. 246, IAU Colloq. 183, Small Telescope Astronomy on Global Scales, ed. B. Paczynski, W.-P. Chen, \& C. Lemme (San Francisco, CA: ASP), 121
Gal-Yam, A., et al. 2004, ApJ, 609, L59

Hill, G. J., Nicklas, H. E., MacQueen, P. J., Mitsch, W., Wellem, W., Altmann, W., Wesley, G. L., \& Ray, F. B. 1998, Proc. SPIE, 3355, 433

Holtzman, J. A., Burrows, C. J., Casertano, S., Hester, J. J., Trauger, J. T., Watson, A. M., \& Worthey, G. 1995, PASP, 107, 1065

Hook, I. M., Jørgensen, I., Allington-Smith, J. R., Davies, R. L., Metcalfe, N., Murowinski, R. G., \& Crampton, D. 2004, PASP, 116, 425

Jeffery, D. J., \& Branch, D. 1990, in Supernovae, Jerusalem Winter School for Theoretical Physics, ed. J. C. Wheeler, T. Piran, \& S. Weinberg (Singapore: World Scientific), 149

Kasliwal, M. M., \& Kulkarni, S. R. 2010, ATel, 2590, 1

Kennicutt, R. C., Jr, et al. 2003, PASP, 115, 928

Khan, R., Stanek, K. Z., Prieto, J. L., Kochanek, C. S., Thompson, T. A., \& Beacom, J. F. 2010, ApJ, 715, 1094

Kulkarni, S. R., \& Kasliwal, M. M. 2009, in Astronomy, Vol. 2010, astro2010: The Astronomy and Astrophysics Decadal Survey, 165

Kulkarni, S. R., et al. 2007, Nature, 447, 458

Law, N. M., et al. 2009, PASP, 121, 1395

Lejeune, T., \& Schaerer, D. 2001, A\&A, 366, 538

Li, W. D., et al. 2000, in AIP Conf. Ser. 522, Cosmic Explosions: Tenth Astrophysics Conf., ed. S. S. Holt \& W. W. Zhang (Melville, NY: AIP), 103

Martin, D. C., et al. 2005, ApJ, 619, L1

Martini, P., Wagner, R. M., Tomaney, A., Rich, R. M., della Valle, M., \& Hauschildt, P. H. 1999, AJ, 118, 1034

Metzger, B. D., Piro, A. L., Quataert, E., \& Thompson, T. A. 2009, MNRAS, 396, 1659

Metzger, B. D., et al. 2010, MNRAS, 406, 2650

Moriya, T., Tominaga, N., Tanaka, M., Nomoto, K., Sauer, D. N., Mazzali, P. A., Maeda, K., \& Suzuki, T. 2010, ApJ, 719, 1445

Morrissey, P., et al. 2007, ApJS, 173, 682

Nugent, P. E. 2009, BAAS, 41, 419

Ofek, E. O., et al. 2008, ApJ, 674, 447

Oke, J. B., et al. 1995, PASP, 107, 375

Pennypacker, C. R., et al. 1989, AJ, 97, 186

Prieto, J. L., et al. 2008, ApJ, 681, L9

Rahmer, G., Smith, R., Velur, V., Hale, D., Law, N., Bui, K., Petrie, H., \& Dekany, R. 2008, Proc. SPIE, 7014, 163

Rau, A., et al. 2009, PASP, 121, 1334

Russell, D. G. 2002, ApJ, 565, 681

Schlegel, D. J., Finkbeiner, D. P., \& Davis, M. 1998, ApJ, 500, 525

Shen, K. J., Kasen, D., Weinberg, N. N., Bildsten, L., \& Scannapieco, E. 2010, ApJ, 715, 767

Sheth, K., et al. 2010, PASP, 122, 1397

Smith, N., et al. 2009, ApJ, 697, L49

Thompson, T. A., Prieto, J. L., Stanek, K. Z., Kistler, M. D., Beacom, J. F., \& Kochanek, C. S. 2009, ApJ, 705, 1364

van Dokkum, P. G. 2001, PASP, 113, 1420

Wright, E. L., et al. 2010, AJ, 140, 1868 